\title{
Pediatric to adult inflammatory bowel disease transition: the Asian experience
}

\author{
Bernice Tan, David Ong \\ Division of Gastroenterology and Hepatology, National University Hospital, Singapore
}

\begin{abstract}
Many tertiary inflammatory bowel disease (IBD) centers recognize that a structured transition program is fundamental for an IBD unit. However, the path to ensuring a seamless transition for all stakeholders is often fraught with challenges. In this review, we go through current evidence, identify the requirementsof a successful transition program, and the barriers to seamless transfer. We also aim to shed light on differences in needs between the Western and Asian adolescent IBD populations. Majority of healthcare providers viewed having a structured transition program to be very important. The lack of a standard protocol led us to come up with a list of requirements ofa successful program. These include: multidisciplinary team meetings, alternating visits between the adultand pediatric clinics, proper documentation of records, and determining the ideal timing for transfer. The difficulties forthe Asian adolescent IBD population may be attributed to the reliance on parental support to make decisions regarding medicaltreatment. Lastly, there are various physician, patient and disease factors which are barriers to seamless transition. These includethe lack of proper documentation, and lack of patient self-efficacy. We also propose a standardized template for documentation of medical records for IBD patients. (Intest Res 2020;18:11-17)
\end{abstract}

Key Words: Inflammatory bowel disease; Transition; Pediatric; Asian; Transitional care

\section{INTRODUCTION}

The age of presentation of IBD is increasingly more common under the age of 16 years. ${ }^{1,2}$ IBD presenting at a young age is also more extensive and severe than in adults. ${ }^{3}$ Hence, it is of utmost importance to ensure proper transition of care to the adult gastroenterologist.

In the context of healthcare, transition is defined as the purposeful, planned movement of adolescents with chronic physical and medical conditions to adult-orientated healthcare systems. $^{4}$

Transition of care for patients with IBD is commonly viewed as a period fraught with challenges for the patients, caregivers

Received November 5, 2019. Revised December 11, 2019.

Accepted December 13, 2019.

Correspondence to Bernice Tan, Division of Gastroenterology and

Hepatology, National University Hospital, 5 Lower Kent Ridge Road,

Singapore 119074. Tel: +65-6772-2002, Fax: +65-6772-4361, E-mail:

bernice_tan@nuhs.edu.sg and healthcare providers alike. Many studies have shown the efficacy of a standardized transition program, ${ }^{5,6}$ and hence major organizations such as the European Crohn's and Colitis Organisation (ECCO) $)^{7}$ do recommend a transition program for every adolescent. Moreover, ECCO states that IBD patients taking part in a transition program are likely to have better compliance and less adverse outcomes after transfer to adult care compared with those who do not take part. However, there is a lack of clear guidelines on the specific recommendations for a standardized transition program.

The authors believe that this may be attributed to the differences in the needs and expectations of IBD patients worldwide. Adolescence is a precarious period for growth and change, and the adolescent must shed the sheltered environment of childhood and achieve self-reliance and self-dependent living. Coupled with cultural differences and extent of parental involvement in patients' care, it is evident that various societies require different levels of intensity for a transition program. Pediatric and adult gastroenterology clinics also have different 
areas of focus that changes with each stage of the patient's life. For example, pediatric clinics may be better able to deal with adolescent issues such as growth and puberty, exam pressure, sexual or recreational drug experimentation, whereas adult gastroenterologists are better geared towards tackling adult issues of fertility, family planning and surveillance of secondary conditions (e.g., malignancy and osteoporosis). Hence, it is fundamental that patients receive the appropriate care at each stage.

This paper is a review of the various studies that have been performed thus far, and aim to look into the basic requirements of a successful transition program. We also aim to identify the barriers to seamless transfer, with special mention of characteristics that are particular to the Asian population.

\section{METHODS}

A literature search on PubMed and Google Scholar was conducted with the keywords "IBD," "transition," "pediatric," "transitional care" and "transition clinic." This search brought up a total of 37 relevant articles. The authors then went through the articles and summarized the pertinent points in this review.

\section{PERCEPTION OF DOCTORS, CAREGIVERS AND PATIENTS ON TRANSITION}

Many of the studies reviewed had polls on medical providers about their perception of transition care. It is not surprising that majority viewed a structured transition program to be very important. ${ }^{5}$ However, a majority of medical providers also did not have a defined protocol for transition. ${ }^{5}$ We find that having a structured transition program is beneficial to the stakeholders involved in the various ways as elaborated on in the points mentioned below.

Adolescents also require more attention during the transition period. A retrospective case-control study ${ }^{3}$ compared disease characteristics and compliance in 100 adolescents with adult controls matched for disease duration. All adolescents were seen at a biweekly transition clinic of IBD patients ages 16-24 years old. A greater percentage of adolescents required hospital admission ( $46 \%$ compared to $14 \%$ of adults, $P<0.0001$ ), azathioprine ( $46 \%$ compared with $17 \%$ of adults, $P<0.0001$ ), and infliximab ( $20 \%$ compared with $6 \%$ of adults, $P<0.05)$. Adolescents missed a median number of $20 \%$ of their appointments compared with $0 \%$ in adults $(P<0.0001)$. This study shows that adolescents have more severe disease that may be due to primary noncompliance or the disease characteristics itself.

\section{REQUIREMENTS OF A SUCCESSFUL TRANSITION PROGRAM}

The following is an outline of the structured program that we propose for transition.

\section{Multidisciplinary Team Meetings}

Transition to self-management involves gradual changes in knowledge, attitudes, and behavior. This requires a multidisciplinary team (including pediatric and adult gastroenterologists, surgeons, parents/caregivers, school workers, nurses, dieticians, counselors, social workers, psychologists, healthcare systems, pharmacists, etc.) whose roles are all essential for seamless continuation of an adolescent's healthcare. Recognition of the roles of these individuals in multidisciplinary care and maximization of communication among them is of strategic importance.

These meetings should be scheduled on a regular basis, and not only in the short months leading up to the planned transfer of care for identified patients. At our center, team meetings between the pediatric and adult IBD teams are scheduled regularly on a 1 to 2 monthly basis. These meetings not only provide a proper place and avenue for discussion of transition cases, but also serve as a time for education and discussion of challenging pediatric and adult IBD cases. Furthermore, since the adult gastroenterologist will eventually take over the care of the patient, it makes sense for him/her to get involved early on in the management of these pediatric patients.

\section{Alternating Visits between the Pediatric and Adult Clinic before Official Transfer}

The aim of these alternating visits is to encourage patient autonomy and individual decision making while still providing some amount of handholding in the familiar background of the pediatric clinic. However, the final visit should be held in the adult gastroenterology clinic, but with the pediatric IBD nurse present.

We would like to emphasize on the importance of having a specialized IBD nurse in this transition process. The IBD nurse is the familiar face for patients and caregivers, and they also provide an invaluable resource for patients to contact should they have additional questions that were not addressed in the clinic. At our center, our IBD nurse is in charge of the IBD hot- 
line-an easy mode of contact for patients, especially if they are experiencing high burden of symptoms or are dealing with adverse effects of therapy. It has also potentially avoided unnecessary inpatient admissions and unscheduled clinic visits.

An Israeli group consisting of an IBD tertiary referral center in Tel Aviv conducted a study ${ }^{6}$ on 36 patients (mean age, $19 \pm$ 1.8 years) found that standardized transition clinics helped to improve self-efficacy scores in the "IBD-yourself" questionnaire. Their transition protocol included an average of 3 to 4 meetings over $6.9 \pm 3.5$ months. Self-efficacy scores in all domains of the questionnaire were significantly higher after completion of the transition.

During these visits, we propose that the IBD team, together with the patient and his/her caregiver, come up with a written healthcare transition plan that is updated with each clinic consult. Leung et al. ${ }^{8}$ proposed a checklist of knowledge and tasks that adolescents should master before they transition fully to an adult care clinic. These include: (1) diagnosis (CD, UC, indeterminate colitis); (2) anatomic location of their disease; (3) extraintestinal manifestations; (4) year of diagnosis, and names of their IBD institutions/hospital/healthcare providers; (5) dates of hospitalizations, why they were hospitalized, events during hospitalizations; (6) dates of surgery and the procedure performed; (7) complications of their disease or therapy, e.g., fistulas, abscesses, opportunistic infections, pancreatitis, cytopenias; (8) names and doses of drugs they are currently on and have taken in the past; (9) insurance plan and contact information; (10) can make arrangements for blood tests and understands who to call for follow-up on the results; (11) calls the appropriate healthcare provider in the event of a disease flare; (12) calls the appropriate healthcare provider to refill medications; (13) can schedule their own visits and remembers to attend; (14) understands which medications can potentially be harmful during pregnancy and the need to discuss with healthcare provider issues regarding contraception and planning for children.

\section{Proper Documentation of Medical Records}

This point is often overlooked by physicians, but it is truly a fundamental component. Organized record keeping allows for easy access and retrieval of medical information, which is crucial for chronic diseases. The management of IBD, in particular, involves multiple lines of treatment-something which the patient's physician needs to be well-aware of what the patient has previously tried or failed before-so that the next line of treatment may be offered. The meticulous tracking of previ- ous endoscopy findings, vaccination records, and lab/stool tests is also important since the follow-up of IBD patients usually go on for many years. We propose the implementation of standardized template for documentation (Fig. 1).

\section{Determining the Ideal Timing for Transition}

The ECCO review suggests that transition is best initiated in early adolescence. ${ }^{7}$ In European countries, the transfer of care is at the age of 18 years, but in the United States, this is often at the age of 21 years or even up to 25 years. ${ }^{9}$ In the Asian context, adulthood may be considered at age 21 years and above.

At the point of transfer of care, it is ideal if the patient were in clinical remission, or at the very least, have only clinically mild disease. During each clinic visit, a medical summary and an emergency plan should be reiterated, especially for patients who are symptomatic of active disease.

We also emphasize the importance of setting goals and deadlines for transfer of care.

\section{DIFFERENCES BETWEEN THE NEEDS OF THE WESTERN AND ASIAN ADOLESCENT IBD POPULATION}

The key difference lies in the different ages that major life events occur for Asian and Western populations. These "major life events" include graduation, entering further education, seeking employment, acquiring financial independence, and moving away from family and home. Many consider these events to be pivotal points in the transition towards adulthood. In the Asian context, these life events tend to occur at later ages. This may be attributed to the traditional Asian values of filial piety and the societal norm of living in a multigenerational household.

According to the various population census, ${ }^{10}$ there are stark differences in terms of ages at which children move out of the parental home. In Hong Kong, 53\% of males and almost 47\% of females aged between 15 and 34 lived with their parents in 2015. Around 29\% of young adults in Australia live with their parents, according to the 2011 census, up from $21 \%$ in 1976 . Some $97 \%$ of unmarried people aged 15 to 34 lived with their parents in Singapore as of 2013, according to the statistics bureau. For married people, it was $37 \%$.

As such, the Asian adolescent is usually thought to be more reliant on parental influence when making decisions. In the context of IBD care, major decisions such as initiating biologic therapy usually tend to be a shared family decision, especially 


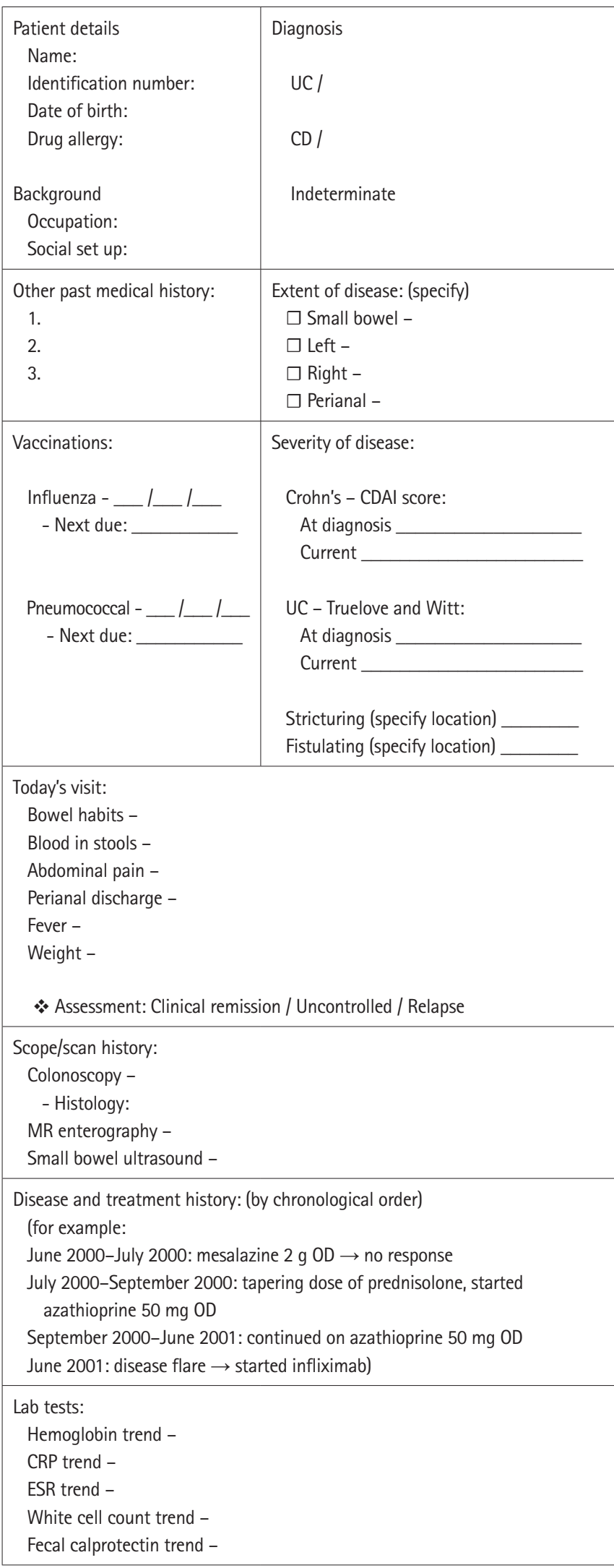

Fig. 1. Proposed template for documentation. MR, magnetic resonance; $\mathrm{OD}$, once daily. since there is a financial consideration with regards to the high cost of treatment. Western culture encourages adolescents to move out of the family home and work their way through college with part-time jobs-but it is not the case in the Asian context where most children still rely on their parents to fund their school fees and living expenses until they enter the workforce.

Therefore, it is essential for the multidisciplinary IBD team to approach this issue differently based on the cultural context that the team practices in. Bearing in mind that finances is one of the main factors that young adults are unable to step away from the financial comfort of their parents/caregivers, the psychologist and social worker in charge of the case should actively engage the adolescent early on. As young IBD patients have the tendency to feel dependent a lot of the time as they have always played the role of the "sick child." Hence, seeking employment and financial independence may not be their priority. It is important for the various stakeholders (such as the IBD nurse, pediatric gastroenterologist and social worker) not to neglect the psychosocial growth of the child as well. However, we must not forget that good control of the underlying disease will lead to better growth of the child and hence less disruption to his/her physical and emotional growth. As such, the child would be able to lead a normal life as much as possible, and be confident in his/her ability to care for and make important medical decisions for themselves. This will subsequently empower them to be mentally and financially self-sufficient, so that the parental role may be reduced.

On the other hand, we should not forget that change can be implemented from the parents/caregivers' side as well. Parents who may potentially be overly protective of their child and hence impede the process of transitioning should be identified early by the healthcare provider so that the multidisciplinary team can make the efforts to effect a change in mindset when the patient is approaching the period of transition. Sometimes, these caregivers may even require emotional counseling from feeling lost after not having a dependent to look after anymore. They may struggle with the sense of wanting to continue taking care of the patient, while on the other hand, wanting the patient to be independent. These feelings may eventually be transferred onto the patient who feels conflicted about wanting to be take charge without negating the appreciation for the parental care that has been provided since childhood. Therefore, the role of the social worker and the psychologist will come in handy in such cases.

We would also like to emphasize that cultures are diverse even amongst various Asian nations. Hence, it is important to 
individualize each transition program according to each country's unique culture and values.

\section{BARRIERS TO A SEAMLESS TRANSFER}

\section{Physician Factors}

\section{1) Lack of Knowledge and/or Experience}

This is especially so for adult physicians who may not be wellversed in the area of adolescent medicine. As we have described above, many adolescent patients are undergoing major changes in their lives at the proposed age of transition of 18 years. This is an understandably delicate period of change when patients have different physical and psychological needs. Adolescents may be going through periods of experimentation in all areas from recreational drugs to cosmetic procedures, and providers with more in-depth knowledge of the intricacies of medical management in relation to these areas of adolescent medicine would be better able to advise patients and their parents.

A survey amongst Japanese adult gastroenterologists also found that physicians in practice for 30 years or more reported less hesitancy in taking over medical care for childhood-onset patients referred from pediatric gastroenterologists. ${ }^{11}$ Hence, the benefit of a team of IBD physicians is that junior physicians are able to continually learn from their more experienced colleagues. This is important as patients and their caregivers need to have confidence in their physician in order to build a trusting relationship that would promote self-efficacy, self-empowerment, and compliance to their medical treatment.

\section{2) Poor Coordination of Care between Adult and Pediatric Providers}

This may be largely attributed to the lack of a specialized IBD nurse who also plays the important role of a transition coordinator. The same Japanese survey identified poor coordination of care and inadequate medical summaries as barriers to smooth transition. ${ }^{11}$

The lack of coordination of care can sometimes also be attributed to logistical factors such as funding, availability of nursing and allied healthcare support, and the patterns of referrals to tertiary centers. In areas with lower socioeconomic status, these factors may ultimately prove to be the largest barrier to a proper transition program.

\section{Patient Factors}

\section{1) Poor Health Literacy/Disease-Specific Knowledge}

Studies have found that physicians tend to overestimate their patients' health literacy-related readiness for transition. ${ }^{12}$ Hence, validated tools may be used to assess functional and interactive health literacy instead. For example, the Test of Functional Health Literacy in Adults ${ }^{13}$ may be used. The 24-item Crohn's and Colitis Knowledge Scale ${ }^{14}$ can also be used to assess patients' interactive health literacy.

\section{2) Lack of Self-Efficacy}

The slow uptake of objective evidence-based assessment tools for measurement of patients' self-efficacy is concerning as physicians may gauge this poorly. In IBD, the most commonly used transition readiness assessment tool is the Transition Readiness Assessment Questionnaire (TRAQ), a 20-item self-report questionnaire regarding disease self-management skills. ${ }^{15}$ However, the TRAQ does not take into account the perspectives of the parent/caregiver and/or the healthcare provider. A potential area for further research would be a comprehensive assessment tool that comprises the parent's and provider's gauge of the patient's readiness to transition as well.

\section{3) High Level of Parental/Caregiver Involvement}

An Ohio group conducted focus group interviews amongst parents/caregivers and found that majority of parents reported that they were heavily involved in their child's IBD care. ${ }^{16}$ This included recognizing when medical care is needed, knowing their child's treatment history, scheduling and attending appointments, answering and asking questions during clinic visits, ordering and picking up refills, and handling financial matters.

The degree of patient independence from their parents/caregivers is crucial in ensuring success for transitional care. The aim is to empower patients with better knowledge about their disease, and also to encourage them to find out more about their condition on their own. This self-empowerment will build a continual cycle of self-interest and a strong desire to seek better control of their IBD. Pediatric gastroenterologists must promote patient independence at regular clinic visits using appropriate tools or programs (e.g., guidelines for autonomy support of patients with IBD in the transitional period ${ }^{17}$ ). High level of parental involvement may serve as a barrier to the development of youth self-management skills.

\section{Disease Factor}

The difficult-to-control disease would inevitably draw out the period of transition as it would be difficult to find an opportune time for transition. Challenging diseases may also be 
contributed in part by the patient/parent's noncompliance to treatment from a young age. These patients would often be labeled as having recalcitrant disease as they are noncompliant to treatment and hence are deemed to have "failed" multiple lines of therapy. Therefore, we cannot emphasize more on the importance of setting strong groundwork from the point of diagnosis so as to ensure good patient compliance and understanding of his/her disease.

\section{CONCLUSION}

Transitional care is increasingly recognized as a mandatory part of the provision of care in any tertiary IBD center. However, there is a lack of evidence-based data on the ideal proposed transition program. Various objective self-assessment tools measuring health literacy and self-efficacy have been validated in the IBD cohort, and we encourage the use of these tools at specialized transition clinics. In conclusion, we have provided a proposed structure for transition based on the experience at our center, and also highlighted the important points to note especially when treading through the different cultural context in the Asian IBD population.

\section{FINANCIAL SUPPORT}

The authors received no financial support for the research, authorship, and/or publication of this article.

\section{CONFLICT OF INTEREST}

No potential conflict of interest relevant to this article was reported.

\section{AUTHOR CONTRIBUTION}

Writing, methodology: Tan B. Conceptualization: Ong D. Approval of final manuscript: all authors.

\section{ORCID}

Tan B https://orcid.org/0000-0003-2860-5768

Ong D https://orcid.org/0000-0002-4953-8042

\section{REFERENCES}

1. Sawczenko A, Sandhu BK, Logan RF, et al. Prospective survey of childhood inflammatory bowel disease in the British Isles. Lancet 2001;357:1093-1094.

2. Lehtinen $\mathrm{P}$, Ashorn M, Iltanen S, et al. Incidence trends of pediatric inflammatory bowel disease in Finland, 1987-2003, a nationwide study. Inflamm Bowel Dis 2011;17:1778-1783.

3. Goodhand J, Dawson R, Hefferon M, et al. Inflammatory bowel disease in young people: the case for transitional clinics. Inflamm Bowel Dis 2010;16:947-952.

4. Blum RW, Garell D, Hodgman CH, et al. Transition from childcentered to adult health-care systems for adolescents with chronic conditions: a position paper of the Society for Adolescent Medicine. J Adolesc Health 1993;14:570-576.

5. Al-Jahdali E, Mosli M, Saadah O. A cross-sectional survey of Saudi gastroenterologists: transition strategies for adolescents with inflammatory bowel disease. Saudi J Gastroenterol 2017; 23:233-237.

6. Zijlstra M, De Bie C, Breij L, et al. Self-efficacy in adolescents with inflammatory bowel disease: a pilot study of the "IBDyourself", a disease-specific questionnaire. J Crohns Colitis 2013;7:e375-e385.

7. Van Rheenen PF, Aloi M, Biron IA, et al. European Crohn's and Colitis Organisation topical review on transitional care in inflammatory bowel disease. J Crohns Colitis 2017;11:1032-1038.

8. Leung Y, Heyman MB, Mahadevan U. Transitioning the adolescent inflammatory bowel disease patient: guidelines for the adult and pediatric gastroenterologist. Inflamm Bowel Dis 2011;17:2169-2173.

9. De Silva PS, Fishman LN. Transition of the patient with IBD from pediatric to adult care: an assessment of current evidence. Inflamm Bowel Dis 2014;20:1458-1464.

10. Nohara Y, Lee J. More adults kept from leaving parents' nests in Asia by harsh economic realities. The Japantimes. https:// www.japantimes.co.jp/news/2016/12/02/national/adultskept-leaving-parents-nests-asia-harsh-economic-realities/\#. XaXK1UYzY2w.

11. Kumagai H, Kudo T, Uchida K, et al. Adult gastroenterologists' views on transitional care: results from a survey. Pediatr Int 2019;61:817-822.

12. Huang JS, Tobin A, Tompane T. Clinicians poorly assess health literacy-related readiness for transition to adult care in adolescents with inflammatory bowel disease. Clin Gastroenterol Hepatol 2012;10:626-632.

13. Parker RM, Baker DW, Williams MV, Nurss JR. The test of functional health literacy in adults: a new instrument for measuring patients' literacy skills. J Gen Intern Med 1995;10:537-541.

14. Eaden JA, Abrams K, Mayberry JF. The Crohn's and Colitis 
Knowledge Score: a test for measuring patient knowledge in inflammatory bowel disease. Am J Gastroenterol 1999;94: 3560-3566.

15. Sawicki GS, Lukens-Bull K, Yin X, et al. Measuring the transition readiness of youth with special healthcare needs: validation of the TRAQ: Transition Readiness Assessment Questionnaire. J Pediatr Psychol 2011;36:160-171.
16. Gray WN, Resmini AR, Baker KD, et al. Concerns, barriers, and recommendations to improve transition from pediatric to adult IBD care: perspectives of patients, parents, and health professionals. Inflamm Bowel Dis 2015;21:1641-1651.

17. Kumagai H, Akiyama T, Abukawa D, et al. Guidelines for autonomy support of patients with IBD in the transitional period. Jpn J Pediatr Gastroenterol Hepatol Nutr 2018;32:15-27. 